\title{
The Uniqueness of the Markoff Numbers
}

\author{
By Gerhard Rosenberger \\ Dedicated to the 60th birthday of Professor Hel Braun
}

\begin{abstract}
A Markoff triple is a set of three positive integers satisfying the diophantine equation $x^{2}+y^{2}+z^{2}=3 x y z$. The maximum of the three numbers is called a Markoff number. We show: If there are Markoff triples $\left(x_{1}, y_{1}, z\right)$ and $\left(x_{2}, y_{2}, z\right)$ with the same Markoff number $z$, then $x_{1}=x_{2}$ or $x_{1}=y_{2}$.
\end{abstract}

A. A Markoff triple is a set of three positive integers satisfying the diophantine equation $x^{2}+y^{2}+z^{2}=3 x y z$. The maximum of the three numbers is called a Markoff number. Here we will prove: If there are Markoff triples $\left(x_{1}, y_{1}, z\right)$ and $\left(x_{2}, y_{2}, z\right)$ with the Markoff number $z$, then $x_{1}=x_{2}$ or $x_{1}=y_{2}$. Some numerical evidence concerning the uniqueness of the Markoff numbers is given in [1] and [4]

Definitions.

$\{A, B\}$ is the group generated by $A$ and $B$.

$[A, B]=A B A^{-1} B^{-1}$ is the commutator of $A, B \in K$ ( $K$ a group). $\operatorname{tr} U$ is the trace of $U \in S L(2, \mathrm{C})$.

B. Lemma 1 (Nielsen [3]). Let $K=\{A, B\}$ be a free group of rank two. Two elements $U, V$ of $K$ generate $K$ if and only if $[U, V]$ is conjugate over $K$ to $[A, B]^{\epsilon}, \epsilon= \pm 1$.

We need the following facts about elements of $\operatorname{SL}(2, \mathrm{C})$ : For all $A, B \in \operatorname{SL}(2, \mathrm{C})$ and $n \geqslant 1$

(a) $\operatorname{tr} A B=\operatorname{tr} A \cdot \operatorname{tr} B-\operatorname{tr} A B^{-1}$.

(b) $\operatorname{tr}[A, B]=(\operatorname{tr} A)^{2}+(\operatorname{tr} B)^{2}+(\operatorname{tr} A B)^{2}-\operatorname{tr} A \cdot \operatorname{tr} B \cdot \operatorname{tr} A B-2$.

(c) $A^{n}=S_{n} A-S_{n-1} I$, where $S_{-1}=-1, S_{0}=0, S_{1}=1, S_{n+1}=(\operatorname{tr} A) \cdot$ $S_{n}-S_{n-1}$.

Now we fix the following notation:

$$
\begin{gathered}
T .=\left(\begin{array}{rr}
0 & 1 \\
-1 & 0
\end{array}\right), \quad R .=\left(\begin{array}{cc}
0 & -1 \\
1 & 1
\end{array}\right), \\
A .=R T R^{2} T=\left(\begin{array}{rr}
1 & -1 \\
-1 & 2
\end{array}\right), \quad B .=T R^{2} T R=\left(\begin{array}{ll}
1 & 1 \\
1 & 2
\end{array}\right) .
\end{gathered}
$$

It is known that

$$
A^{-1}=T R B R^{-1} T^{-1}=R^{-1}(A B) R
$$

Received November 20, 1973.

AMS (MOS) subject classifications (1970). Primary 10B10, 10D05, $20 \mathrm{H} 10$.

Copyright $\odot 1976$, American Mathematical Society 
and $\operatorname{tr}[A, B]=-2$. Because of $\operatorname{tr} A=3$, we have $\operatorname{tr} A^{n}=\operatorname{tr} B^{n}=\operatorname{tr}(A B)^{n}>\operatorname{tr} A^{m}$ for $n>m>0$.

The modular group $G$ is generated by $T$ and $R$, and it is

$$
G=\left\{T, R \mid T^{2}=R^{3}=1\right\} \quad(G=\operatorname{PSL}(2, \mathrm{Z})) .
$$

The commutator group $G^{\prime}=[G, G]$ of $G$ is generated by $A$ and $B$; and $G^{\prime}$ is a free group of rank two. By Lemma 1 we have $(\operatorname{tr} U)^{2}+(\operatorname{tr} V)^{2}+(\operatorname{tr} U V)^{2}=\operatorname{tr} U \cdot \operatorname{tr} V \cdot$ $\operatorname{tr} U V$ for any pair $(U, V)$ of generators of $G^{\prime}$ (see (b)).

LEMma 2. For $n, m, r, s \in \mathbf{N}$ the following facts are true:

(1) $\operatorname{tr} A B^{n}>\operatorname{tr} A B^{m}$ for $n>m$.

(2) $\operatorname{tr} A B^{n}>\operatorname{tr} A B^{r} A B^{s}$ for $n \geqslant 4, n \geqslant r+s$.

(3) $\operatorname{tr} A B^{n}<\operatorname{tr} A B^{r} A B^{s}$ for $r+s>n$.

(4) $\operatorname{tr} A B^{n} A B^{m}>\operatorname{tr} A B^{r} A B^{s}$ for $n+m>r+s$.

Proof. (1) $\operatorname{tr} A B^{n}=\operatorname{tr} A\left(S_{n} B-S_{n-1} I\right)=\operatorname{tr}\left(S_{n} A B-S_{n-1} A\right)=3\left(S_{n}-S_{n-1}\right)$ $>3\left(S_{m}-S_{m-1}\right)=\operatorname{tr} A B^{m}$ for $n>m$.

(2) It is sufficient to prove this for $n=r+s$ and $s=1$, i.e. $n=r+1$, or $s=2$. Let $s=1$. Then, $\operatorname{tr} A B^{r} A B=\operatorname{tr}\left(\left(S_{r} A B-S_{r-1} A\right) A B\right)=\operatorname{tr}\left(S_{r}(A B)^{2}-S_{r-1} A^{2} B\right)=$ $7 S_{r}-6 S_{r-1}<3 S_{n}-3 S_{n-1}=\operatorname{tr} A B^{n}$; because of $n \geqslant 4$. The proof for $s=2$ is analogous.

(3) This is trivial for $r>n$ or $s>n$. Let us consider now $r \leqslant n$ and $s \leqslant n$. It is sufficient to prove this for $r+s=n+1$ and $s=1$, i.e. $n=r . \operatorname{tr} A B^{n} A B=$ $7 S_{n}-6 S_{n-1}>3\left(S_{n}-S_{n-1}\right)=\operatorname{tr} A B^{n}$.

(4) This is trivial for $n>r+s$ or $m>r+s$. Let us consider now $n \leqslant r+s$ and $m \leqslant r+s$. It is sufficient to prove this for $m+n=r+s+1$. Then $m>r$, $m>s, n>r$ or $n>s$; say $m>s$. Now we may assume $s=1$, i.e. $m+n=r+2$.

(a) $n \geqslant r$. Then it is sufficient to prove this for $r=1$; i.e. $m+n=3$. Then $m=2$ because of $m>s$. $\operatorname{tr} A B A B^{2}=15>7=\operatorname{tr} A B A B$.

(b) $r \geqslant n$. Then it is sufficient to prove this for $n=1$, i.e. $m=r+1$; and therefore, we may assume $r=1$, too, i.e. $m=2$. $\operatorname{tr} A B A B^{2}>\operatorname{tr} A B A B$. Q.E.D.

Remark. Some of our main arguments in this proof were, for instance, the following:

Let $n, m, r, s \in \mathbf{N}$.

(1) If $\operatorname{tr} A B^{n} A B^{m}<\operatorname{tr} A B^{r} A B^{s}$ for $n+m<r+s$, then $\operatorname{tr} A B^{n} A B^{m+1}<$ $\operatorname{tr} A B^{r} A B^{s+1}$.

(2) If $\operatorname{tr} A B^{n}<\operatorname{tr} A B^{r} A B^{s}$, then $\operatorname{tr} A B^{n+1}<\operatorname{tr} A B^{r} A B^{s+1}$.

(3) If $\operatorname{tr} A B^{n}>\operatorname{tr} A B^{r} A B^{s}, n \geqslant 4$, then $\operatorname{tr} A B^{n+1}>\operatorname{tr} A B^{r} A B^{s+1}$.

With these and similar arguments, in connection with some suitable conjugations, we can construct the following lemma:

Lemma 3. Let $C_{1}=A B^{\epsilon_{1}} \cdots A B^{\epsilon_{n}}, 2 \leqslant \epsilon_{i}$, and $C_{2}=A B^{\alpha_{1}} \cdots A B^{\alpha_{m}}, 2 \leqslant$ $\alpha_{j}$. Let $k_{1}=n+\sum_{i=1}^{n} \epsilon_{i}=n+s_{1}, k_{2}=m+\sum_{j=1}^{m} \alpha_{j}=m+s_{2}$. Let $s_{1} \geqslant s_{2}$ for $n<m$, respectively, $s_{1}>s_{2}$ for $m \leqslant n$. Then $\operatorname{tr} C_{1}>\operatorname{tr} C_{2}$.

Proof. We prove this lemma inductively over the possible quadruples $\left(s_{1}, s_{2}\right.$, $n, m)$, where the quadruples $\left(s_{1}, s_{2}, n, m\right)$ are ordered by: 


$$
\left(s_{1}^{\prime}, s_{2}^{\prime}, n^{\prime}, m^{\prime}\right)<\left(s_{1}, s_{2}, n, m\right) \rightleftharpoons s_{1}^{\prime} \leqslant s_{1}, \quad s_{2}^{\prime} \leqslant s_{2}, \quad n^{\prime} \leqslant n, \quad m^{\prime} \leqslant m
$$

and

$$
s_{1}^{\prime}+s_{2}^{\prime}+n^{\prime}+m^{\prime}<s_{1}+s_{2}+n+m
$$

For suitable small quadruples $\left(s_{1}, s_{2}, n, m\right)$ the statement is true by Lemma 2 .

Let $\left(s_{1}, s_{2}, n, m\right)$ be a possible quadruple. We assume the statement is true for all possible quadruples $\left(s_{1}^{\prime}, s_{2}^{\prime}, n^{\prime}, m^{\prime}\right)$ with $\left(s_{1}^{\prime}, s_{2}^{\prime}, n^{\prime}, m^{\prime}\right)<\left(s_{1}, s_{2}, n, m\right)$.

Case 1. $\epsilon_{i}=2$ for $i=1, \ldots, n$. Then $C_{1}=\left(A B^{2}\right)^{n}, n \geqslant 2$ and $n>m$, i.e. $s_{1}>s_{2}$ and $k_{1}>k_{2}$. If $m \geqslant 2$, then it follows by assumption that $\operatorname{tr} A B^{s_{2}}>\operatorname{tr} C_{2}$. Therefore, the statement is true, if we can show $\operatorname{tr} C_{1}>\operatorname{tr} A B^{s_{2}}$.

Obviously, the statement is true for $s_{1}>s_{2}$, if we can show it for $2 n=s_{1}=s_{2}$ +1 . And we get

$$
\begin{aligned}
\operatorname{tr}\left(A B^{2}\right)^{n} & =\operatorname{tr}\left(A B^{2}\right) \cdot S_{n}\left(\operatorname{tr} A B^{2}\right)-2 S_{n-1}\left(\operatorname{tr} A B^{2}\right) \\
& =6 S_{n}(6)-2 S_{n-1}(6)>3\left(S_{2 n-1}(3)-S_{2 n-2}(3)\right) \\
& =3\left(S_{2 n-1}(\operatorname{tr} B)-S_{2 n-2}(\operatorname{tr} B)\right)=\operatorname{tr} A B^{2 n-1} \quad \text { by induction. }
\end{aligned}
$$

Case 2. $\alpha_{j}=2$ for $j=1, \ldots, m$. Then $C_{2}=\left(A B^{2}\right)^{m}$.

Obviously, the statement is true for $m \leqslant n$.

Let us consider now $n<m$. Then $\epsilon_{i} \geqslant 3$ for some $i$. Obviously, the statement is true for $s_{1} \geqslant s_{2}$, if we can show it for $s_{1}=s_{2}=2 \mathrm{~m}$.

Let us consider now $s_{1}=s_{2}$. For $n=m-1$ the statement is true by direct calculations. Let us consider now $n<m-1$. It is $\operatorname{tr}\left(A B^{2}\right)^{m-2} A B^{4}>\operatorname{tr}\left(A B^{2}\right)^{m}$; and therefore, it follows by assumption that

$$
\operatorname{tr} C_{1}>\operatorname{tr}\left(A B^{2}\right)^{m-2} A B^{4}>\operatorname{tr} C_{2} .
$$

Case 3. $\epsilon_{i} \geqslant 3$ for some $i$ and $\alpha_{j} \geqslant 3$ for some $j$. We may assume, perhaps after suitable conjugations, $\epsilon_{n} \geqslant 3$ and $\alpha_{m} \geqslant 3$. Let

$$
C_{1}^{\prime}=A B^{\epsilon_{1}} \cdots A B^{\epsilon_{n}-1}, \quad C_{2}^{\prime}=A B^{\alpha_{1}} \cdots A B^{\alpha_{m}-1} .
$$

Then $\operatorname{tr} C_{1}^{\prime}>\operatorname{tr} C_{2}^{\prime}$ implies by a simple calculation

$$
\operatorname{tr} C_{1}=\operatorname{tr} C_{1}^{\prime} B>\operatorname{tr} C_{2}^{\prime} B=\operatorname{tr} C_{2} \text {. Q.E.D. }
$$

C. THEOREM. Let $\left(x_{1}, y_{1}, z\right)$ and $\left(x_{2}, y_{2}, z\right)$ be Markoff triples with the same Markoff number $z$. Then $x_{1}=x_{2}$ or $x_{1}=y_{2}$ (and therefore, $y_{1}=y_{2}$ or $y_{1}=x_{2}$ ).

Proof. If a triple $(x, y, z)$ of three positive integers is a solution of the diophantine equation $x^{2}+y^{2}+z^{2}=x y z$, then $x, y, z \equiv 0(\bmod 3)$, i.e.: With the integral solutions of $x^{2}+y^{2}+z^{2}=x y z$ we have also the integral solutions of $x^{\prime 2}+y^{\prime 2}+$ $z^{\prime 2}=3 x^{\prime} y^{\prime} z^{\prime}$ and conversely. Therefore, the theorem is proved if we can show: If $\left(x_{1}, y_{1}, z\right)$ with $x_{1}, y_{1} \leqslant z$ and $\left(x_{2}, y_{2}, z\right)$ with $x_{2}, y_{2} \leqslant z$ are triples of positive integers satisfying the diophantine equation $x^{2}+y^{2}+z^{2}=x y z$, then $x_{1}=x_{2}$ or $x_{1}=y_{2}$.

Let $\left(x_{1}, y_{1}, z\right)$ with $x_{1}, y_{1} \leqslant z$ and $\left(x_{2}, y_{2}, z\right)$ with $x_{2}, y_{2} \leqslant z$ be triples of 
positive integers satisfying the diophantine equation $x^{2}+y^{2}+z^{2}=x y z$. The theorem is certainly true for $x_{1}=z, x_{2}=z, y_{1}=z$ or $y_{2}=z$.

Let us consider now $x_{1}, y_{1}, x_{2}, y_{2}<z$. Especially, $z>3$. By [2] and [5] there are generators $\left(A_{1}, B_{1}\right)$ and $\left(A_{2}, B_{2}\right)$ of the commutator group $G^{\prime}$ of the modular group $G$ with

(1) $\operatorname{tr} A_{1}=z, \operatorname{tr} B_{1}=x_{1}, \operatorname{tr} A_{1} B_{1}=y_{1}$, and

(2) $\operatorname{tr} A_{2}=z, \operatorname{tr} B_{2}=x_{2}, \operatorname{tr} A_{2} B_{2}=y_{2}$.

Moreover, $\operatorname{tr}\left[A_{1}, B_{1}\right]=\operatorname{tr}\left[A_{2}, B_{2}\right]=-2$. By [2, Theorem 2.1], we may assume that $A_{i}$ is conjugate over $G^{\prime}$ to an element $M_{r_{i}}, s_{i}=\Pi_{j=1}^{r i} A B^{a_{i j}+2}$ or its inverse, where $\left(r_{i}, s_{i}\right)$ an integer pair with $r_{i}>0, s_{i} \geqslant 0,\left(r_{i}, s_{i}\right)=1$ and $a_{i j}=\left[j s_{i} / r_{i}\right]-$ $\left[(j-1) s_{i} / r_{i}\right] \quad(i=1,2)$. By Lemma 3 we have $r_{1}=r_{2}, s_{1}=s_{2}$ and $a_{1 j}=a_{2 j}$ (here $\operatorname{tr} A_{1}=\operatorname{tr} A_{2}$ ); that means we may assume that $A_{1}$ is conjugate over $G^{\prime}$ to $A_{2}$ or its inverse. Now with regard to Lemma 1 and $(*)$ we may assume, perhaps after a suitable conjugation,

(a) $A_{2}=A_{1}^{\alpha}, \alpha= \pm 1$, and

(b) $\left[A_{1}, B_{1}\right]=\left[A_{1}^{\gamma}, B_{2}^{\delta}\right]$ or $\left[A_{1}, B_{1}\right]=\left[B_{2}^{\delta}, A_{1}^{\gamma}\right] ; \gamma, \delta= \pm 1$.

Case 1. Let $\left[A_{1}, B_{1}\right]=\left[B_{2}^{\delta}, A_{1}^{\gamma}\right]$. Then we have necessarily $\gamma=-1$, because otherwise

$$
B_{2}^{\delta} A_{1} B_{2}^{-\delta}=A_{1} B_{1} A_{1}^{-1} B_{1}^{-1} A_{1} \quad \text { and } \quad z=\operatorname{tr} A_{1}=z \cdot \operatorname{tr}\left[A_{1}, B_{1}\right]-z=-3 z .
$$

We get $A_{1}=B_{1}^{-1} A_{1}^{-1} B_{2}^{\delta} A_{1} B_{2}^{-\delta} A_{1} B_{1}$; i.e. $B_{1}^{-1} A_{1}^{-1} B_{2}^{\delta}$ and $A_{1}$ commute. Therefore, $B_{1}^{-1} A_{1}^{-1} B_{2}^{\delta}$ and $A_{1}$ have the same fixed points. Since the commutator subgroup $G^{\prime}$ of the modular group is free, we have $B_{2}^{\delta}=A_{1} B_{1} A_{1}^{\beta}$. Assume $\beta \geqslant 1$. Then

$$
x_{2}=\operatorname{tr} B_{1} A_{1}^{\beta+1}=y_{1} S_{\beta+1}-x_{1} S_{\beta}=\left(y_{1} z-x_{1}\right) S_{\beta}-y_{1} S_{\beta-1}>z,
$$

and that is not true. Therefore, $\beta \leqslant 0$. Assume $\beta \leqslant-2$. Then $x_{2}=\operatorname{tr} B_{1} A_{1}^{\beta+1}=$ $x_{1} \cdot \operatorname{tr} A_{1}^{-\beta-1}-\operatorname{tr} B_{1} A_{1}^{-\beta-1}=\left(x_{1} z-y_{1}\right) S_{-\beta-1}>z$, and that is not true. Therefore, $\beta=0$ or $\beta=-1$. We have $x_{2}=y_{2}$ for $\beta=0$ and $x_{2}=x_{1}$ for $\beta=-1$.

Case 2. Let $\left[A_{1}, B_{1}\right]=\left[A_{1}^{\gamma}, B_{2}^{\delta}\right]$. Then we have necessarily $\gamma=1$, because otherwise again $z=-3 z$. We get $A_{1}=B_{1}^{-1} B_{2}^{\delta} A_{1} B_{2}^{-\delta} B_{1}$, i.e., $B_{1}^{-1} B_{2}^{\delta}$ and $A_{1}$ commute. Therefore $B_{1}^{-1} B_{2}^{\delta}$ and $A_{1}$ have the same fixed points. Since $G^{\prime}$ is free, we have $B_{2}^{\delta}=$ $B_{1} A_{1}^{\beta}$. Assume $\beta \geqslant 2$. Then

$$
x_{2}=\operatorname{tr} B_{1} A_{1}^{\beta}=y_{1} S_{\beta}-x_{1} S_{\beta-1}=\left(y_{1} z-x_{1}\right) S_{\beta-1}-y_{1} S_{\beta-2}>z,
$$

and that is not true. Therefore, $\beta \leqslant 1$. Assume $\beta \leqslant-1$. Then $x_{2}=\operatorname{tr} B_{1} A_{1}^{\beta}=$ $x_{1} \operatorname{tr} A_{1}^{-\beta}-\operatorname{tr} B_{1} A_{1}^{-\beta}=\left(x_{1} z-y_{1}\right) S_{-\beta}>z$, and that is not true. Therefore, $\beta=0$ or $\beta=1$. We have $x_{2}=x_{1}$ for $\beta=0$ and $x_{2}=y_{1}$ for $\beta=1$. This completes the proof. Q.E.D.

Mathematisches Seminar der Universität 
1. I. BOROSH, "Numerical evidence on the uniqueness of Markoff numbers," Notices Amer. Math. Soc., v. 21, 1974, p. A-55. Abstract \#711-10-32.

2. H. COHN, "Markoff forms and primitive words," Math. Ann., v. 196, 1972, pp. 8-22. MR $45 \# 6899$.

3. J. NIELSEN, "Die Isomorphismen der allgemeinen unendlichen Gruppe mit zwei Erzeugenden," Math. Ann., v. 78, 1918, pp. 385-397.

4. D. ROSEN \& G. S. PATTERSON, JR., "Some numerical evidence concerning the uniqueness of the Markov numbers," Math. Comp., v. 25, 1971, pp. 919-921. MR 46 \#132.

5. G. ROSENBERGER, “Fuchssche Gruppen, die freies Produkt zweier zyklischer Gruppen sind, und die Gleichung $x^{2}+y^{2}+x^{2}=x y z$," Math. Ann., v. 199, 1972, pp. 213-227. MR 49 \#5202. 